\title{
Rapid notes
}

\section{$\mu^{+}$SR studies of $\mathrm{UM}_{2} \mathrm{Al}_{3}, \mathrm{M}=\mathrm{Ni}, \mathrm{Pd}$}

\author{
A. Amato ${ }^{1}$, C. Geibel $^{2}$, F.N. Gygax ${ }^{1}$, R.H. Heffner ${ }^{3}$, E. Knetsch ${ }^{4}$, D.E. MacLaughlin ${ }^{4,5}$, C. Schank ${ }^{2}$, A. Schenck ${ }^{1}$, \\ F. Steglich ${ }^{2}$, and M. Weber ${ }^{1}$ \\ ${ }^{1}$ Institut für Mittelenergiephysik der ETH Zürich, CH-5232 Villingen PSI, Switzerland \\ ${ }^{2}$ Institut für Festkörperphysik, Technische Hochschule, W-6100 Darmstadt, Federal Republic of Germany \\ ${ }^{3}$ Los Alamos National Laboratory, Los Alamos, NM 87545, USA \\ ${ }^{4}$ Kamerlingh Onnes Laboratory, Rijksuniversiteit Leiden, 2300 RA Leiden, The Netherlands \\ ${ }^{5}$ Physics Department, University of California, Riverside, CA 92521-0413, USA
}

Received October 7, 1991

Positive-muon spin rotation $\left(\mu^{+} \mathrm{SR}\right)$ measurements have been carried out in the new heavy-fermion superconductors $\mathrm{UM}_{2} \mathrm{Al}_{3}, \mathrm{M}=\mathrm{Ni}$ and $\mathrm{Pd}$. In $\mathrm{UNi}_{2} \mathrm{Al}_{3}$ the observed $\mu^{+}$frequencies in zero applied field indicate commensurate antiferromagnetic (AF) ordering of $U$ moments $\sim 0.1 \mu_{B}$ /U atom. These moments coexist with superconductivity and have the highest values observed in an AF heavy-fermion superconductor. The absence of well-defined frequencies in zero-field $\mu^{+} \mathrm{SR}$ in the AF state of $\mathrm{UPd}_{2} \mathrm{Al}_{3}$ suggests symmetric $\mu^{+}$stopping sites. In this system $\mu^{+} \mathrm{SR}$ linewidths below $T_{c}$ yield a preliminary value of $\sim 8000 \AA$ for the London penetration depth.

Heavy-fermion superconductivity has recently been reported in the homologous ternary compounds $\mathrm{UM}_{2} \mathrm{Al}_{3}, \mathrm{M}=\mathrm{Ni}[1]$ and $\mathrm{Pd}$ [2]. In $\mathrm{UNi}_{2} \mathrm{Al}_{3}$ a transition to an antiferromagnetic (AF) state is observed at a Néel temperature $T_{N} \simeq 4.5 \mathrm{~K}$, followed by a superconducting transition at $T_{c} \simeq 1 \mathrm{~K}$ [1]. The corresponding transition temperatures in $\mathrm{UPd}_{2} \mathrm{Al}_{3}$ are $T_{N}$ $\simeq 14 \mathrm{~K}$ and $T_{c} \simeq 2 \mathrm{~K}$, respectively [2]; the latter is the highest value observed to date for a heavy-fermion superconductor.

This Rapid Note reports results of positive-muon $\left(\mu^{+}\right)$spin rotation $\left(\mu^{+} \mathrm{SR}\right)$ experiments in $\mathrm{UNi}_{2} \mathrm{Al}_{3}$ and $\mathrm{UPd}_{2} \mathrm{Al}_{3}$ which yield information on the AF spin structure and superconductivity in these compounds. Our principal conclusions are the following:

(1) Zero-field $\mu^{+} \mathrm{SR}$ frequencies in $\mathrm{UNi}_{2} \mathrm{Al}_{3}$ are consistent with a simple commensurate AF structure. The zero-temperature sublattice magnetization derived from these frequencies, $\sim 0.1 \mu_{B} / \mathrm{U}$ atom, is the largest value found to date in an $\mathrm{AF}$ heavy-fermion superconductor. The AF order coexists with and is not appreciably affected by superconductivity below $T_{c} \sim 1 \mathrm{~K}$. (2) In $\mathrm{UPd}_{2} \mathrm{Al}_{3}$, in contrast to $\mathrm{UNi}_{2} \mathrm{Al}_{3}$, no well-defined frequencies appear in zero-field $\mu^{+} \mathrm{SR}$ for $T<T_{N}$. This suggests near cancellation of local fields at $\mu^{+}$stopping sites, which must therefore be symmetric with respect to the AF sublattices in this material. Transverse-field $\mu^{+} \mathrm{SR}$ linewidths in the superconducting state of $\mathrm{UPd}_{2} \mathrm{Al}_{3}$ yield a London penetration depth $\sim 8000 \AA$ and no indication of unconventional superconducting pairing.

Polycrystalline samples were prepared and characterized as described previously $[1,2]$. The $\mu^{+} \mathrm{SR}$ experiments were carried out at the Low Temperature $\mu$ SR Facility at the Paul Scherrer
Institute, Villigen, Switzerland. A representative zero-field histogram for $\mathrm{UNi}_{2} \mathrm{Al}_{3}$ together with the fit to a four-component depolarization function is shown in Fig. 1. In the following the four components and their associated fit parameters will be labeled 1 through 4 . Component 1 is best fit by a static Kubo-Toyabe function [3] appropriate to a site with a Gaussian distribution of local fields with zero mean. Components 2,3 , and 4 are best fit by exponentially-damped sinusoids. Such well-defined $\mu^{+} \mathrm{SR}$ frequencies indicate a commensurate AF structure.

The low-temperature frequencies, relaxation rates, asymmetries (amplitudes), and site assignments (using the Wyckoff-notation $[4,5]$, see below) of each of these components are given in Table 1. The asymmetries of the precessing components (Components 2, 3, and 4) have been corrected by a factor $3 / 2$ to take into account the random orientations of the internal fields in our polycrystalline sample. The temperature dependences of the frequencies are given in Fig. 2. It can be seen that (a) to good accuracy each frequency follows a spin-1/2 Brillouin function with $T_{N}=4.35 \mathrm{~K}$, and (b) there is no measurable effect of the superconducting transition at $\sim 1 \mathrm{~K}$.

The multi-component nature of the $\mu^{+} \mathrm{SR}$ signal in $\mathrm{UNi}_{2} \mathrm{Al}_{3}$ indicates several $\mu^{+}$stopping sites in the hexagonal $\operatorname{PrNi}_{2} \mathrm{Al}_{3}$ structure. Similar $\mu^{+} \mathrm{SR}$ signals have been observed in $\operatorname{PrNi}_{5}$ [4], which has a related crystal structure.

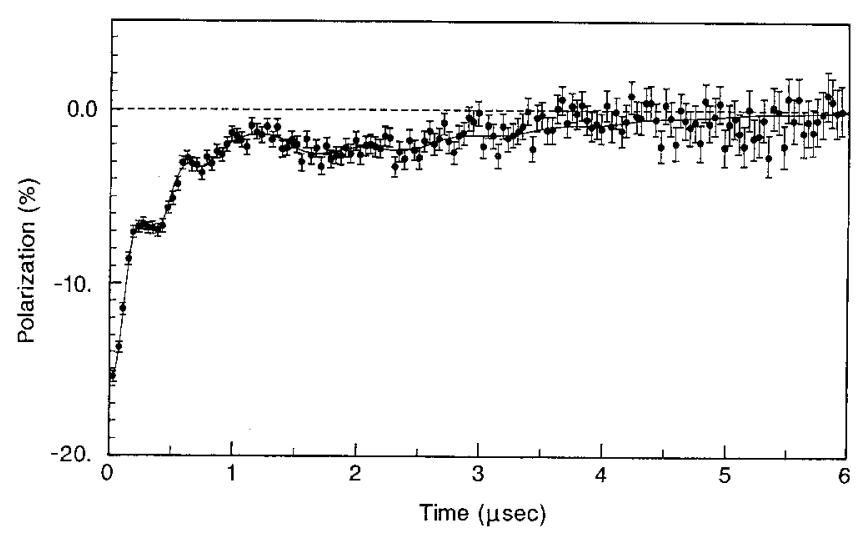

Fig. 1. Zero-field $\mu^{+} \mathrm{SR}$ histogram for $\mathrm{UNi}_{2} \mathrm{Al}_{3}, T=0.3 \mathrm{~K}$. Solid curve: bestfit four-component depolarization function 
Table 1. Parameters for four-component fits to zero-field $\mu^{+}$SR histograms $\left(T \ll T_{N}\right)$ in $\mathrm{UNi}_{2} \mathrm{Al}_{3}$

\begin{tabular}{|c|c|c|c|c|c|}
\hline $\begin{array}{l}\text { Compo- } \\
\text { nent }\end{array}$ & $\begin{array}{l}\text { Fit } \\
\text { Function }\end{array}$ & $\begin{array}{l}\text { Frequency } \\
(\mathrm{MHz})\end{array}$ & $\begin{array}{l}\text { Relax. } \\
\text { rate } \\
\left(\mu \mathrm{s}^{-1}\right)\end{array}$ & $\begin{array}{l}\text { Asymmetry } \\
(\%)\end{array}$ & $\begin{array}{l}\text { Site } \\
\text { assignment } \\
\text { (no. sites/ } \\
\text { unit cell) }\end{array}$ \\
\hline 1 & $\mathrm{~K}-\mathrm{T}^{\mathbf{a}}$ & 0 & $0.27^{\mathrm{a}}$ & 3.05 & $b(1)$ \\
\hline 2 & exp. & 0.46 & $1.7^{\mathrm{b}}$ & $12.2^{\mathrm{c}}$ & $h(4)$ \\
\hline $\begin{array}{l}3 \\
4\end{array}$ & exp. & 1.26 & 1.0 & \multirow{2}{*}{\multicolumn{2}{|c|}{$\left.\begin{array}{l}1.2 \\
7.95\end{array}\right\} 9.15^{\mathrm{c}, \mathrm{d}}$}} \\
\hline 4 & exp. & 2.6 & 3.5 & & \\
\hline
\end{tabular}

a Zero-field Kubo-Toyabe relaxation function [3]

b Exponential relaxation function

- Corrected by a factor $3 / 2$ (see text)

d Components 3 and 4 fractionally assigned to Site $f$ (see text)

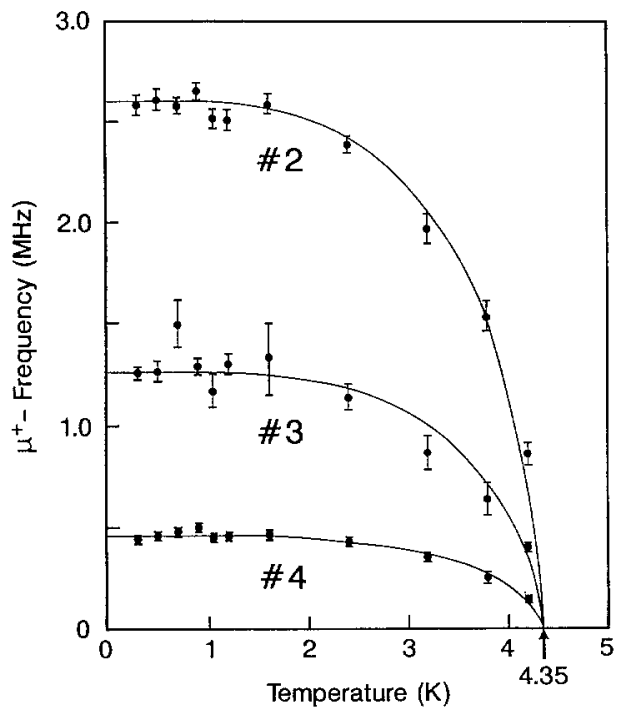

Fig. 2. Temperature dependence of zero-field $\mu^{+} \mathrm{SR}$ frequencies in $\mathrm{UNi}_{2} \mathrm{Al}_{3}$. Solid curves: spin- $1 / 2$ Brillouin functions with $T_{N}=4.35 \mathrm{~K}$

As criteria for site assignment we have required that the relative numbers of the assigned sites accord with the relative asymmetries of the corresponding components; and that Component 1 , with zero frequency, be due to muons at a symmetric site with respect to the AF sublattices, at which the dipolar or transferred hyperfine fields from $U$ moments cancel. A secondary criterion was the observed occupation of candidate sites in materials with similar structures either by hydrogen (e.g., in $\mathrm{LaNi}_{5} \mathrm{H}_{6}$ [5]) or by muons (e.g., in $\mathrm{PrNi}_{5}$ [4]): this was fulfilled by the site assignments described below.

Using these criteria we have identified the most probable $\mu^{+}$stopping sites as $b, h$, and $f$ for Components 1,2 , and $(3+4)$, respectively, with the proviso that Components 3 and 4 are both associated with the $f$ site even though the two frequencies $v_{3}$ and $v_{4}$ are different $\left(v_{3} / v_{4} \simeq 1 / 2\right)$. Although the reason for this difference is not understood, no other choice of site assign- ment could account for the observed asymmetries and the value of the Kubo-Toyabe relaxation rate for Component 1 . In any event the asymmetry of Component 3 is small $(\sim 4 \%$ of the total asymmetry).

With these site assignments very good agreement is found between numbers of sites and relative component asymmetries. The calculated value of the Kubo-Toyabe relaxation rate [3] due to ${ }^{27} \mathrm{Al}$ nuclear dipolar fields at site $b, 0.192 \mu \mathrm{s}^{-1}$, is smaller than the observed value of $0.27 \mu \mathrm{s}^{-1}$. This would be expected in the presence of slight structural disorder, which would destroy the perfect cancellation of the AF sublattice fields. No other candidate site obeys this inequality and exhibits a zero AF local field for reasonable AF structures.

Using the above site assignments, $\mu^{+}$AF local fields were calculated assuming ferromagnetic alignment in hexagonal $\mathrm{U}$ basal planes and $\mathrm{AF}$ alignment between planes. The ratio $v_{4} / v_{2} \simeq 5$ is reproduced only for these site assignments and for $\mathrm{U}$ moments directed along the $c$ axis. Assuming this structure, the observed frequencies yield a static $U$ moment of $\sim 0.12 \mu_{B}$ /U atom.

Preliminary $\mu^{+}$SR measurements have been also carried out in $\mathrm{UPd}_{2} \mathrm{Al}_{3}$ at temperatures below $T_{N}$. The $\mu^{+} \mathrm{SR}$ behavior in this compound is very different from that in $\mathrm{UNi}_{2} \mathrm{Al}_{3}$. In zero applied field only a zero frequency signal was observed, similar to Component 1 in $\mathrm{UNi}_{2} \mathrm{Al}_{3}$, implying that only site $b$ is occupied in $\mathrm{UPd}_{2} \mathrm{Al}_{3}$

An incommensurate AF structure could also account for the absence of well-defined frequencies, but not for the weak depolarization of the zero frequency signal, which displays a decay rate similar to Component 1 in $\mathrm{UNi}_{2} \mathrm{Al}_{3}$.

In transversely applied fields the $\mu^{+} \mathrm{SR}$ signal in $\mathrm{UPd}_{2} \mathrm{Al}_{3}$ exhibits additional broadening in the superconducting state and a negative shift of the $\mu^{+}$precession frequency due to diamagnetic screening. Preliminary analysis of the additional broadening yields low-temperature behavior consistent with conventional superconducting Cooper pairing, and a zero-temperature London penetration depth $\lambda \simeq 8000 \AA$. This is within a factor of two of previous indirect estimates [2].

We are grateful to C. Baines, D. Herlach, and the accelerator crew of PSI for their expert help during the experiments. This work was supported by the Deutsche Forschungsgemeinschaft under the auspices of the Sonderforschungsbereich 252 Darmstadt/Frankfurt/Mainz, the U.S. Department of Energy, the Netherlands NWO and FOM, and the University of California, Riverside, Academic Senate Committee on Research.

\section{References}

1. Geibel, C., Thies, S., Kaczorowski, D., Mehner, A., Grauel, A., Seidel, B., Ahlheim, U., Helfrich, R., Petersen, K., Bredl, C.D., Steglich, F.: Z. Phys. B - Condensed Matter 83, 305 (1991)

2. Geibel, C., Schank, C., Thies, S., Kitazawa, H., Bredl, C.D., Böhm, A., Rau, M., Grauel, A., Caspary, R., Helfrich, R., Ahlheim, U., Weber, G., Steglich, F.: Z. Phys. B - Condensed Matter 84, 1 (1991)

3. For a description of the $\mu$ SR technique see Schenck, A.: Muon Spin Rotation Spectroscopy. Adam Hilger, Bristol, 1985

4. Hitti, B., Birrer, P., Grayersky, A., Gygax, F.N., Kaplan, N., Lippelt, N., Schenck, A., Weber, M.: Hyperfine Interact. 59, 377 (1990)

5. Lartigue, C., Percheron-Guegan, A., Achard, J.C., Soubeyroux, J.L.: J. Less-Common Met. 113, 127 (1985) 